\title{
Nontuberculous mycobacteria - clinical and laboratory diagnosis: experiences from a TB endemic country
}

\author{
Ann Susan Sam*,1(i), Marilyn Mary Ninan'1, R Ranjani', Naveen Kumar Devanga \\ Raghupathi $^{1}$ (D), Veeraraghavan Balaji ${ }^{1}$ (i) \& Joy Sarojini Michael ${ }^{1}$ \\ ${ }^{1}$ Department of Clinical Microbiology, Christian Medical College, Vellore-632004, India \\ *Author for correspondence: annsam1736@gmail.com
}

\begin{abstract}
Aim: To evaluate the performance of VITEK ${ }^{\circledR}$ MS with DNA sequencing for laboratory diagnosis of nontuberculous mycobacteria (NTM) species in a resource-limited setting. Methods: 16SrRNA sequencing and MALDI-TOF mass spectrometry (VITEK ${ }^{\circledR}$ MS) was performed at a tertiary-care hospital in India. MALDI-TOF results were confirmed by $16 \mathrm{~S}$ rRNA sequencing. In addition, sequencing of the internal transcribed spacer region was performed on slowly growing NTM. Results: Commonest species isolated were $M$. abscessus, M. intracellulare, M. avium, M. fortuitum and $M$. simiae. $16 \mathrm{~S}$ rRNA sequencing and MALDI-TOF results had agreement of $94.5 \%$ for rapidly growing and $77.5 \%$ for slowly growing NTM. Conclusion: There is good correlation between VITEK ${ }^{\circledR}$ MS and sequencing for rapidly growing NTM. For slowly growing species, sequencing would be required in a third isolates.
\end{abstract}

First draft submitted: 13 February 2020; Accepted for publication: 26 May 2020; Published online: 30 July 2020

Keywords: geographic distribution • identification • MALDI-TOF mass spectrometry • nontuberculous mycobacteria - prevalence • sequencing • VITEK ${ }^{\circledR} \mathrm{MS}$

The nontuberculous mycobacteria (NTM) are described as Mycobacteria other than Mycobacterium tuberculosis complex and Mycobacterium leprae [1]. There are more than 190 species/subspecies of NTM [2], which were previously considered as environmental organisms of limited clinical relevance. The AIDS epidemic in the $1980 \mathrm{~s}$ brought a dramatic increase in the numbers of NTM infection, particularly due to those of Mycobacterium avium complex. The use of iatrogenic immunosuppression has also added to the burden of disease [3,4]. The spectrum of disease caused by NTM can be classified into four groups - progressive pulmonary disease, cervical lymphadenitis, skin and soft tissue infection and disseminated. Disseminated infections are typically seen only in immunocompromised individuals [5]. Identification of NTM species is extremely important, treatment of these infections are species specific [6].

\section{Species identification of NTM}

Biochemical tests are only useful in the detection of the most common 10-15 species and have a prolonged turnaround time. Hence, newer methods such as high-performance liquid chromatography (HPLC), line probe assay (LPA), DNA probes, matrix-assisted laser desorption ionization-time of flight (MALDI-TOF) and DNA sequencing have been developed. HPLC, LPA and commercial DNA probes have limited utility as they are restricted to identification of the most frequently isolated NTM species. The two MALDI-TOF mass spectrometry (MS) platforms, namely VITEK ${ }^{\circledR}$ MS (bioMérieux, Marcy-l'Étoile, France) and Bruker Daltonics platform (Bremen, Germany) for NTM identification, have been evaluated to identify NTM from both solid and liquid media. VITEK $^{\circledR}$ MS is a rapid, cost effective and reliable platform, which has the capability to identify as many as 24 NTM species $[7,8]$. This technology is designed to provide a protein 'fingerprint' based on the desorbed ions from cell surfaces by measuring their mass-to-charge ratio. The instrument's software automatically acquires and analyzes the data and generates a profile for comparison with a database of reference spectra, composed of previously well-

FUTURE SCIENCE 
characterized isolates, providing the species identification of all the common and some of the uncommon NTM species [9].

The latest in vitro diagnostic (IVD) database (V3.2.0 Knowledge Base) of VITEK ${ }^{\circledR}$ MS allows for better discrimination between species and diversity among strains within the same species [7]. The Spectral Archiving and Microbial Identification System v 4.12 is a specialized research use only library for VITEK ${ }^{\circledR}$ MS, containing 1286 spectra from 45 Mycobacterium species [10].

The latest version of the Bruker Daltonics platform, also known as MBT Mycobacteria library version 6.0, contains spectra for 178 of the currently known 201 mycobacteria species [11].

Sequencing of the $16 \mathrm{~S}$ ribosomal RNA gene (rRNA), which is approximately 1500 base pairs, is the most widely used sequencing method for the identification of bacteria and, is considered as the reference standard for identification of NTM species. It identifies both new and rare species, which cannot be identified by existing methods. Region $\mathrm{A}$, at the $5^{\prime}$ end, contains the majority of the species-specific or 'signature sequences' in mycobacteria [12]. However, there are many species that cannot be identified by $16 \mathrm{~S}$ rRNA sequence alone. For example, M. gastri and M. kansasii sequevars - I \& IV - harbor same 16S sequence [13].

Although, another target for DNA sequencing is the internal transcribed spacer (ITS) sequence, which separates the 16S and 23S rRNA genes and is denominated ITS 1. The sequence of this fragment comprises of 200-330 base pairs and thus, can easily be analyzed. Several sets of primers that enable the amplification and sequencing of the complete fragment have been published. For the ITS 1 sequence, the high variability utilized for species identification has been documented. Roth et al. demonstrated that in situations where $16 \mathrm{~S}$ rRNA gene sequences were indistinguishable, such as for M. kansasii and M. gastri, or highly similar, such as for M. malmoense and $M$. szulgai, the 16S-23S rRNA gene ITS sequences were a helpful supplement for the differentiation of closely related species. As with the $16 S r R N A$ gene sequence, M. marinum and M. ulcerans have identical ITS sequences, thus, cannot be differentiated utilizing this analysis [14,15].

It is important to speciate the NTMs to allow physicians to provide the appropriate treatment and to avoid giving unnecessary treatment for species that has not been associated with clinical significance [6]. The objective of this study was to evaluate the performance of MALDI-TOF by VITEK ${ }^{\circledR}$ MS against Sanger sequencing of 16S rRNA and ITS regions.

\section{Materials \& methods}

This was a prospective study conducted over a period of 24 months from January 2017 to December 2018 at the Department of Clinical Microbiology, Christian Medical College, (Vellore, India), which is a tertiary care hospital that caters to patients from various parts of the country and from neighboring countries, particularly Bangladesh. 90 NTM culture isolates from pulmonary (39 sputum, 8 bronchoalveolar lavage, 2 lung biopsy and 1 endotracheal aspirate) and extrapulmonary ( 3 exudates, 9 blood cultures, 4 bone marrow aspirates, 4 tissue biopsies, 3 lymph node biopsies, 3 urine, 2 corneal scraping and 2 catheter tip) samples grown on Lowenstein Jensen medium were included, in accordance to the inclusion and exclusion criteria described below.

\section{Case definitions}

A diagnosis of NTM lung disease was made by isolation of NTM from at least two separate expectorated sputum samples, or a single specimen if it was collected aseptically from a sterile body site such as endotracheal aspirate, bronchoalveolar lavage or lung tissue. To rule out contamination or probable colonization, laboratory results were correlated with the individual's clinical presentation and radiological and histological findings [16].

A case of extrapulmonary NTM infection was defined as having $>1$ isolate from skin/soft tissue (wound, abscess, tissue or exit catheter); disseminated sites (blood, bone marrow, cerebrospinal fluid, pericardial fluid or peritoneal fluid); lymph node; joint (synovial tissue or joint fluid); or other sites (urine, eye, sinus or nasopharyngeal) $[17,18]$.

\section{MALDI-TOF}

Protein extraction was performed using the VITEK ${ }^{\circledR}$ MS Mycobacteria/Nocardia kit. The protocol provided by the manufacturer was followed. Well isolated colonies of NTM from Lowenstein Jensen media were added to a tube with glass beads and ethanol. It was homogenized utilizing a bead beater, followed by inactivation. Formic acid and acetonitrile were added to the lysate. For each organism to be tested, the supernatant was transferred onto the VITEK ${ }^{\circledR}$ MS-DS slide spots (bioMerieux, NC, USA). VITEK ${ }^{\circledR}$ MS CHCA matrix ( $\alpha$-cyano-4-hydroxycinnamic acid) was added to each sample spot. For instrument calibration, an Escherichia coli reference strain (ATCC 8739) 
was transferred using $1 \mu$ loop to designated spot. Target slides were analyzed by VITEK ${ }^{\circledR}$ MS mass spectrometer IVD (version 3.0) database [19].

\section{Sequencing}

Two to three loopfuls (loop of $10 \mu \mathrm{m}$ diameter) of well-isolated NTM colonies were emulsified in $0.9 \%$ saline. DNA extraction was performed utilizing QIAamp ${ }^{\circledR}$ DNA mini kit (Qiagen, Hilden, Germany) with a 1-h preliminary bacterial cell lysis step using lysozyme buffer, followed by DNA quantification using NanoDrop ${ }^{T M} 2000$ spectrophotometer (Thermo Fisher Scientific, Inc, MA, USA). The extracted product was used for sequencing. The forward and reverse primers used for 16SrRNA sequencing were $5^{\prime}$-AGAGTTTGATCCTGGCTCAG- $3^{\prime}$ and 5'-ACGGTTACCTTGTTACGACTT-3', respectively [20]. The thermal profile of $16 \mathrm{~S}$ rRNA amplification was as follows: initial denaturation for $15 \mathrm{~min}$ at $95^{\circ} \mathrm{C}$, followed by 30 cycles of denaturation at $95^{\circ} \mathrm{C}$ for $1 \mathrm{~min}$, annealing at $52^{\circ} \mathrm{C}$ for $30 \mathrm{~s}$ and extension at $72^{\circ} \mathrm{C}$ for $1.5 \mathrm{~min}$. The final extension phase was at $72^{\circ} \mathrm{C} \mathrm{for} 10 \mathrm{~min}$. The forward and reverse primers used for ITS 1 sequencing were $5^{\prime}$-GTGGGATCGGCGATTGGGAC- $3^{\prime}$ and 5'-CCACCATGCGCCCTTAGACAC-3', respectively [21]. The cycling condition for ITS 1 sequencing was initial denaturation for $95^{\circ} \mathrm{C}$ for $5 \mathrm{~min}$, followed by 38 cycles of $95^{\circ} \mathrm{C}$ for $1 \mathrm{~min}, 55^{\circ} \mathrm{C}$ for $30 \mathrm{~s}$ and $72^{\circ} \mathrm{C}$ for $1 \mathrm{~min}$ and final extension phase of $72^{\circ} \mathrm{C}$ for $10 \mathrm{~min}$. Sequencing was performed using the ABI PRISM BigDye Terminator Cycle Sequencing kit V3.1 (Applied Biosystems ${ }^{\top M}$, CA, USA) and was carried out on the ABI 3500 Genetic Analyzer (Applied Biosystems ${ }^{\top M}$ ). The resulting nucleotide sequences were aligned and Basic Local Analysis Search Tool (BLAST) matched against the reference sequences in the National Center for Biotechnology Information (NCBI; MD, USA) database.

\section{Data analysis}

The clinical and demographic data were analyzed by using the SPSS version 20.0 (IBM, NY, USA) and Microsoft Excel (MS Office 2016) software.

\section{Results}

\section{Prevalence of NTM}

During this 2-year study period, 81,777 samples were received for mycobacterial culture, of which 2775 (3.39\%) were culture positive for mycobacteria; among them, 170 (6.12\%) were identified as NTM.

\section{Geographic distribution}

The patients included in this study came from different states of India, Bangladesh and Nepal.

\section{Clinical profiles of the isolates}

Fifty of the NTM isolates included in this study (55.5\%) were from pulmonary specimens, including sputum, bronchoalveolar lavage, lung biopsy and endotracheal aspirate. 40 isolates were from extrapulmonary specimens such as wound discharges, blood, bone marrow, tissue biopsy, lymph node biopsy, urine, corneal scraping and catheter tip. 49 (54\%) were slowly growing NTM and 41 (46\%) were rapidly growing NTM. The slowly growing NTM predominantly caused pulmonary infections and disseminated infections in HIV seropositive individuals. Among the 49 patients who presented with NTM pulmonary disease, 40 (81.6\%) were due to slowly growing NTM, with the predominant species being identified as M. intracellulare $(17 / 40,42.5 \%)$. Twenty five patients with pulmonary disease had past history of tuberculosis $(25 / 50=50 \%)$ and 20 of them had associated bronchiectasis $(20 / 50=40 \%)$.

Disseminated infection was the second most common presentation of slowly growing NTM (7/90, 7.77\%). $M$. avium, $M$. intracellulare and M. simiae were isolated from bone marrow and lymph node specimens. Six out of these seven patients had AIDS, with a $\mathrm{CD}_{4}$ cell count less than $150 \mathrm{cells} / \mu \mathrm{l}$ at the time of diagnosis. The median $\mathrm{CD}_{4}$ count of these patients was 124 cells/ $\mu$ l. One of the patients who presented with disseminated disease was a 3-year-old child with severe combined immunodeficiency disorder (SCID) from whom M. avium was isolated from tissue biopsy of the middle ear.

However, rapidly growing NTM's produced a wider spectrum of infections - particularly skin and soft tissue infections - with postoperative wound infections being the most common (Table 2). 
Table 1. Geographic distribution of the study population whose isolates were included in the study.

\begin{tabular}{|ll|}
\hline Geographic area & Number of cases, percentage \\
\hline Tamil Nadu, India & $25 / 90,28 \%$ \\
\hline West Bengal, India & $23 / 90,23 \%$ \\
\hline Anarkhand, India & $12 / 90,13 \%$ \\
\hline Kerala, India & $10 / 90,11$ \\
\hline Bangladesh & $6 / 90,7 \%$ \\
\hline Bihar, India & $5 / 90,6 \%$ \\
\hline Chhattisgarh, India & $4 / 90,4 \%$ \\
\hline Karnataka, India & $2 / 90,2 \%$ \\
\hline Nepal & $2 / 90,2 \%$ \\
\hline
\end{tabular}

\section{Table 2. Clinical presentations of rapidly growing nontuberculous mycobacteria.}

\begin{tabular}{ll|} 
Clinical presentation & Number of cases, percentage \\
\hline Skin and soft tissue infections & $16 / 41,39$ \\
\hline Blood stream/line associated infections & $9 / 41,21.9$ \\
\hline Pulmonary infections & $9 / 41,21.9$ \\
\hline Injection site abscesses & $2 / 41,4.8$ \\
\hline Infections of the urinary system & $2 / 41,4.8$ \\
\hline Breast abscess & $1 / 41,2.4$ \\
\hline Skull base osteomyelitis & $1 / 41,2.4$ \\
\hline Chemotherapy port site infection & $1 / 41,2.4$ \\
\hline
\end{tabular}

Species identification by sequencing

Sequencing of the $16 \mathrm{~S}$ rRNA (reference standard) was used to confirm the species identification obtained by MALDI-TOF. The most common slowly growing NTMs isolated were $M$. intracellulare (19 isolates) followed by M. simiae (8 isolates), M. avium (6 isolates), M. avium complex (3 isolates), M. kansasii (2 isolates), M. europaeum (2 isolates), M. szulgai (1 isolate), $M$. yongonense (1 isolate), $M$. timonense (1 isolate), M. scrofulaceum (1 isolate), $M$. parascrofulaceum (1 isolate) and $M$. longobardum (1 isolate). Species with intermediate growth rate such as $M$. flavescens ( 2 isolates) and M. novocastrense (1 isolate) have been included along with slowly growing NTM's in this study. The most common rapidly growing NTM isolated were $M$. abscessus (26 isolates) followed by $M$. fortuitum (10 isolates), M. farcinogenes (1 isolate), M. bacteremicum (1 isolate), M. mucogenicum (1 isolate), M. phocaicum (1 isolate) and $M$. goodii (1 isolate).

ITS sequencing was performed on all the slowly growing NTM. Out of the six Mycobacterium avium isolates, all were identified as $M$. avium subspecies hominisuis by ITS sequencing. The results were compared with 16S rRNA sequencing and MALDI-TOF as described in the Table 3. 16S rRNA and ITS sequencing had an agreement of 95.5\% (95\% CI: 82.4-99.7\%). MALDI-TOF and ITS sequencing of slowly growing NTM demonstrated 76\% agreement (95\% CI: 63.9-81.4\%).

Species identification by matrix-assisted laser desorption ionization mass spectrometry MALDI-TOF using VITEK ${ }^{\circledR}$ MS was evaluated against the aforementioned DNA sequencing methods. MALDITOF identified 76 of the 90 isolates up to species or complex/group level. However, it could not identify 12 $(12 / 90,13.3 \%)$ isolates. Two isolates were misidentified as non-mycobacterial species, in other words, M. goodii as Staphylococcus equorum and $M$. phocaicum as Pseudomonas stutzeri). Species that were not identified by MALDI-TOF included $M$. intracellulare, $M$. abscessus, $M$. avium complex, $M$. yongonense, $M$. longobardum and $M$. scrofulaceum (Table 3).

The statistical agreement between the species identification by MALDI-TOF, with the reference standard 16S rRNA sequencing, is described in Table 4. 


\begin{tabular}{|c|c|c|}
\hline \multirow{2}{*}{$\begin{array}{l}\text { Species-based on } 16 \mathrm{~S} \text { rRNA } \\
\text { sequencing }\end{array}$} & \multirow{2}{*}{$\begin{array}{l}\text { ITS sequencing } \\
\text { Number of concordant isolates }\end{array}$} & \multirow{2}{*}{$\begin{array}{l}\text { MALDI-TOF ID } \\
\text { Number of concordant isolates }\end{array}$} \\
\hline & & \\
\hline M. intracellulare $(n=19)$ & 19 & 14 ( 5 were not identified) \\
\hline M. simiae $(n=8)$ & 8 & 8 \\
\hline M. avium $(n=6)$ & 6 & 5 ( 1 was not identified) \\
\hline M. avium complex $(n=3)$ & 3 (identified as $M$. intracellulare) & 2 (1 was not identified) \\
\hline M. kansasii $(n=2)$ & 2 & 2 \\
\hline M. flavescens $(n=2)$ & 2 & 2 \\
\hline M. europaeum $(n=2)$ & 2 & $\begin{array}{l}0 \text { ( } 1 \text { was identified as } M . \text { gordonae, } 1 \text { was not identified as it was not included in the } \\
\text { database) }\end{array}$ \\
\hline M. szulgai $(n=1)$ & 1 & 1 \\
\hline M. yongonense $(n=1)$ & 1 & Not identified as it was not included in the database \\
\hline M. timonense $(n=1)$ & 1 & 0 (M. avium complex) \\
\hline M. novocastrense $(n=1)$ & 0 (M. flavescens) & 0 (M. flavescens) \\
\hline M. scrofulaceum $(n=1)$ & 0 (M. parascrofulaceum) & 0 (no identification) \\
\hline M. parascrofulaceum $(n=1)$ & 1 & Not included in the database \\
\hline M. longobardum $(n=1)$ & 0 (M. celatum) & Not included in the database \\
\hline M. abscessus $(n=26)$ & $\dagger$ & 24 ( 2 were not identified) \\
\hline M. fortuitum $(n=10)$ & $\dagger$ & 10 (M. fortuitum group) \\
\hline M. farcinogenes $(n=1)$ & $\dagger$ & 1 \\
\hline M. bacteremicum $(n=1)$ & $\dagger$ & 0 (M. neoaurum) \\
\hline M. mucogenicum $(n=1)$ & $\dagger$ & 1 \\
\hline M. phocaicum $(n=1)$ & $\dagger$ & 0 (Pseudomonas stutzeri) \\
\hline M. goodii $(n=1)$ & $\dagger$ & Not included in the database \\
\hline
\end{tabular}

Table 4. Agreement between the results of 16S rRNA sequencing, ITS sequencing and MALDI-TOF.

\begin{tabular}{|c|c|c|}
\hline $\begin{array}{l}\text { Tests compared } \\
\text { (Total tests, } n=90 \text { ) }\end{array}$ & Statistical agreement & $95 \% \mathrm{Cl}$ \\
\hline Concordance of $16 \mathrm{~S}$ rRNA sequencing and MALDI-TOF & $83 \%$ (cumulative) & $74.00-90.4 \%$ \\
\hline - Rapidly growing NTM & $94.5 \%$ & $81.3-99.4 \%$ \\
\hline - Slowly growing NTM & $77.5 \%$ & $63.9-81.4 \%$ \\
\hline Concordance of ITS sequencing for slowly growing & $76 \%$ & $63.9-81.4 \%$ \\
\hline
\end{tabular}

NTM and MALDI-TOF

ITS: Internal transcribed spacer; MALDI-TOF: Matrix assisted laser desorption ionization-time of flight; NTM: Non-tuberculous mycobacteria.

\section{Discussion}

According to the American Thoracic Society (ATS; NY, USA)/Infectious Diseases Society of America (IDSA; VA, USA) statement of 2007, "clinically significant NTM isolates should be routinely identified to the species level" [16]. The British Thoracic Society (London, UK) guidelines in 2017 also recommends that NTM should be identified to the species level utilizing validated molecular or mass spectrometry techniques, as treatment of NTM infections are species-specific and therefore, pathogens must be distinguished from environmental contaminants [6]. Thus, timely and accurate identification of NTM is required to guide therapy.

\section{Prevalence of NTM}

A study on the retrospective analysis of the isolation rates of NTM from various clinical specimens was conducted at this institute during the period of 1999-2004 [22]. The prevalence of NTM at that time period was 3.9\%. The current study demonstrated the prevalence of NTM as $6.12 \%$, which corresponds to the increase in the numbers of NTM globally. However, data from the South Korean national healthcare insurance data identified the age-adjusted prevalence as 33.3 cases / 100,000 population during 2003-2016 [23]. The lack of a nation-wide registry for NTM cases in India is probably reflected in the lower prevalence rate noted in this study. 


\begin{abstract}
Geographic distribution
The study was performed at a tertiary referral center in South India. The study population was from the Indian states and neighboring countries as shown in Table 1 . The climatic conditions of Indian states vary widely and the relationship between climate and the occurrence of NTM infections has not been studied across the country to date. However, NTM is ubiquitously present in the environment and can colonize the human epithelia, which could lead to opportunistic infections, particularly wound infections if the healthcare facilities are inadequate [24-26].
\end{abstract}

\title{
Role of other lung diseases in the occurrence of NTM
}

We observed that the pulmonary NTM infections often occurred in the individuals with a history of tuberculosis and bronchiectasis, although it is well established that lung damage due to previous tuberculosis is a risk factor for NTM pulmonary disease [27].

\section{NTM infections \& associated immunodeficient states}

Of the seven patients with disseminated NTM infection, six were diagnosed with AIDS, with a median $\mathrm{CD}_{4}$ count of 124 cells/cu.mm, which was higher than the other previously reported studies conducted in the patients with disseminated NTM infections, which determined a median CD4 count of 10 cells/cu.mm. This may be due to the higher prevalence of NTM in our environment and the healthcare setting [28-30].

\section{Spectrum of clinical features}

The most common clinical manifestation of patients with rapidly growing NTM were skin and soft tissue infections - predominantly due to postoperative wound infections. NTM bacteremia with rapidly growing NTM was seen in seven patients with prolonged hospitalization of more than 2 weeks, who had central venous line in situ, had undergone invasive procedures of the cardiovascular system (stent, valve repair) or were undergoing therapy for hematological malignancies or immune system disorders. This finding correlates with the studies from other centers in USA [31].

\section{Species-wise distribution}

M. abscessus was the most common species identified during the study period, followed by M. intracellulare (Table 3). We observed that there were no $M$. chelonae isolates identified during the study period, which is in contrast with a previous study conducted in this hospital, which identified 46\% NTM isolates as $M$. chelonae [22]. The number of $M$. simiae cases are also on the rise in the study population. The NTM-Network European Trials Group (NET; Borstel, Germany) conducted a multicentric study in 2008 to find the relative distribution of the different NTMs in pulmonary samples. Mycobacterium avium complex (MAC) predominated in most countries (47\%), followed by M. gordonae (11\%). M. xenopi and M. gordonae had a high rate of isolation in Europe. Isolates from Asia (Japan, South Korea and Taiwan) were predominantly MAC (54\%) followed by a significant proportion of rapid growers (31\%) [32]. In contrast to this data, a study from North India by Umrao et al. determined that the slowly growing NTMs were isolated more frequently (59\%) when compared with rapidly growing species ( $41 \%$ of isolates) [33].

\section{Molecular methods \& NTM species identification}

In this study, complete sequencing of the 16S rRNA gene of 90 NTM isolates was performed. A percentage similarity of $99-99.9 \%$ or more was regarded as the cut-off for the identification of the most accurate species as per the CLSI MM18-A, 2008 guidelines [34]. The most common species identified were M. abscessus, M. intracellulare, M. fortuitum. M. simiae and M. avium, which is similar to the studies from around the globe. However, in a high burden low resource country like India, complete $16 \mathrm{~S}$ rRNA sequencing can only be performed at referral laboratories and is not cost effective to be performed on every isolate.

Three isolates, which were identified as $M$. avium complex by $16 \mathrm{~S}$ rRNA sequencing, were found to be $M$. intracellulare on ITS sequencing. The ITS 1 sequence has a high variability and can be used for species discrimination, especially with slowly growing species including MAC [15]. In this study, ITS sequencing was performed on all slowly growing NTM isolates. All the MAC isolates were identified up to the species level, while $M$. avium was identified up to subspecies level. Out of the $6 \mathrm{M}$. avium isolates, all were identified as $M$. avium subspecies hominisuis, which is the most pathogenic subspecies to humans according to various studies [35]. Rare species such as $M$. yongonense and $M$. timonense can be identified using this method. Hence, ITS sequencing can 
be utilized as an additional method over 16S rRNA sequencing for identification of slowly growing, as well as novel/rare NTM species.

\section{MALDI-TOF MS \& NTM species identification}

The accuracy of this method is dependent upon obtaining good quality spectra. This can be challenging due to the complex cell wall of Mycobacteria which needs specialized protein extraction kits.

In this study, isolates were tested using the VITEK ${ }^{\circledR} \mathrm{MS}$ system, and the results were analyzed using the VITEK ${ }^{\circledR}$ MS IVD version 3.0 database. As the results have demonstrated, there are gaps to be filled in the accurate species-level identification of NTM. 13 out of the 90 isolates (14.4\%) were not given any genus or species-level identification and 2 were misidentified $(2 / 90,2 \%)$ as nonmycobacterial species. Of the 13 isolates that were not identified, 3 species were not in the database, namely M. europaeum, M. yongonense and M. longobardum. However, some of the M. intracellulare (5 out of 19 isolates), M. abscessus ( 2 out of 28 isolates), MAC, M. scrofulaceum and $M$. avium isolates were not identified even though they were included in the database. $M$. timonense, $M$. parascrofulaceum, M. bacteremicum and $M$. farcinogenes were identified as M. intracellulare, M. scrofulaceum, $M$. neoaurum and $M$. fortuitum group, respectively. The studies that evaluated the MALDI TOF MS platforms for the NTM identification also highlighted similar results - for instance 7/244 [3\%] and 2/244 [1\%] isolates were misidentified by the Biotyper system and the VITEK ${ }^{\circledR}$ MS system, respectively [36].

Although MALDI-TOF by VITEK ${ }^{\circledR}$ MS has been successful in the identification of many species of NTM, it cannot distinguish several clinically significant species and subspecies, including $M$. abscessus subsp. abscessus, $M$. abscessus subsp. massiliense and M. abscessus subspecies, M. bolletii; M. chelonae and M. abscessus; M. intracellulare and M. chimaera; $M$. mucogenicum and M. phocaicum; subspecies of $M$. avium and M. abscessus; and species within the $M$. fortuitum group. In spite of good quality spectrum, if there is a 'no identification' call, this may indicate that the organism is not represented in the database of the MALDI-TOF MS platform. Newer species are added and databases are regularly updated by manufacturers. In contrast, if the spectral pattern is poor and multiple species identification are given, there may be mixed culture present, and purity of the isolate should be confirmed [36]. In a similar study performed by Luo et al. on the evaluation of VITEK ${ }^{\circledR} \mathrm{MS}$ version 3.0 for NTM identification, $425 / 507(83.8 \%)$ isolates were identified the first time, 51/507 (11.24\%) additional isolates were identified on repeat, $23 / 507(4.53 \%)$ isolates remained unidentified and 8/507 (1.6\%) isolates were misidentified at species level. Among slowly growing NTM, only 2 out of 56 (3.6\%) M. avium and 3 out of 153 (2\%) M. intracellulare were unidentified, which was marginally better than the findings in this study [8]. The concordance of $77.5 \%$ between VITEK ${ }^{\circledR}$ MS and $16 S$ rRNA sequencing in the identification of slowly growing Mycobacteria is predominantly due to the fact that MALDI-TOF requires moderate growth to be present on the solid media for identification, rather than the scant growth required for sequencing, as demonstrated by other studies as well [37]. Therefore, MALDI-TOF needs to be performed initially for all SGMs as it is cost effective and, in those isolates, which have no identification, sequencing will be required.

\section{MALDI-TOF platforms \& methodologies}

There have been various studies in the past few years that have evaluated the various platforms and databases for the species identification of NTM. Overall performance in the identification of NTM clinical isolates utilizing MALDI-TOF MS was comparable between the bioMérieux VITEK ${ }^{\circledR}$ MS and Bruker MALDI Biotyper systems (MA, USA) in a study by Brown-Elliott $e$ t al. [36]. The systems identified $92 \%(225 / 244)$ and $95 \%(231 / 244)$ of all isolates to at least the complex/group level, respectively. The Biotyper system identified 151/244 isolates (62\%) to the highest taxonomic level, either the species or subspecies level, depending on the organism. The VITEK ${ }^{\circledR}$ MS system identified $138 / 244$ of all isolates $(57 \%)$ to the highest taxonomic level. The limitations common to both platforms is that they cannot differentiate among members of $M$. abscessus complex, $M$. fortuitum group, $M$. avium complex and $M$ mucogenicum group. This is evident from this study as well. Both platforms cannot identify $M$. terrae reliably. However, this could not be assessed as there were no $M$. terrae isolates identified by sequencing in this study.

The latest database for clinical use in the VITEK ${ }^{\circledR}$ MS platform, in other words, IVD v. 3.2.0 and the database used in this study (IVD 3.0) has no difference in the species included [19,38].

MALDI-TOF is less expensive per test, after the initial purchase of the mass spectrometry instrument, which makes it a suitable assay for routine diagnostics. Sequencing is five- to six-times more expensive than MALDI-TOF and therefore, should be used as a confirmatory test. MALDI-TOF requires only protein extraction, which is a 
simple technique that can be performed by any laboratory technologist in a routine clinical microbiology laboratory setting. However, sequencing needs trained personnel for the procedure in a molecular laboratory setting. MALDITOF is a rapid method that takes 1.5 to $2 \mathrm{~h}$ for the whole procedure, whereas sanger sequencing takes on average $12-24 \mathrm{~h}[39]$.

\section{Limitations}

The emended genus Mycobacterium encompasses the 'Tuberculosis-Simiae' clade, and four novel genera viz. Mycolicibacterium gen. nov., Mycolicibacter gen. nov., Mycolicibacillus gen. nov. and Mycobacteroides gen. nov. corresponding to the 'Fortuitum-Vaccae', 'Terrae', 'Triviale' and 'Abscessus-Chelonae' clades, respectively. However, the isolates included in this study have continued to use the previous classification as the emended genus names were not available in the VITEK ${ }^{\circledR}$ MS database at the time of study.

The subspecies of MABC (Mycobacterium abscessus complex) differs in their antibiotic susceptibility profiles and disease prognosis. Subspecies-level identification of $M$. abscessus could not be performed using the sequencing of 16S rRNA or MALDI-TOF by VITEK ${ }^{\circledR}$ MS.

Climatic conditions of the geographic area from which the patients come from could not be addressed in this study.

\section{Conclusion}

MALDI-TOF is a relatively novel technology, especially in a resource-limited country. It is an excellent method for diagnostic stewardship for species identification of rapidly growing NTM, as it is simple-to-use, rapid, less expensive (after the initial purchase of the mass spectrometry instrument) and accurate. Targeted gene sequencing may be required for the rare and novel species of NTM, particularly the slowly growing species as the database lacks the rare species. However, gene sequencing needs to be performed using multiple targets for complete identification up to subspecies level, which may be available only in the reference laboratories.

\section{Future perspective}

As infections due to NTM are on the rise, there is a necessity for timely diagnosis and accurate speciation for appropriate patient management. This study has demonstrated that MALDI-TOF mass spectrometry is an essential tool to be integrated into routine clinical microbiology laboratories and DNA sequencing technology in diagnostic laboratories with adequate infrastructure.

\section{Executive summary}

- The prevalence of nontuberculous mycobacteria (NTM) during the study period was $6.12 \%$, which is in agreement with the increase in the numbers of NTM globally.

- The cumulative concordance of MALDI-TOF with the reference standard $16 \mathrm{~S}$ rRNA sequencing was $83.33 \%$.

- MALDI-TOF assay is a rapid and cost-effective method for identification of NTM in a routine diagnostic laboratory, particularly for the rapidly growing species.

- Clinical profile of these isolates was also studied which showed pulmonary infection (55\%) being the most common clinical presentation of slowly growing species and skin and soft tissue infection (56\%) for the rapidly growing species.

- The numbers of invasive infections due to NTM such as Mycobacterium abscessus is on the rise.

- MALDI-TOF is useful for species identification of rapidly growing NTM as it is simple-to-use, rapid, less expensive and accurate.

- VITEK ${ }^{\circledR}$ MS cannot distinguish several clinically significant species and subspecies, including $M$. abscessus subsp. abscessus, $M$. abscessus subsp. massiliense and $M$. abscessus subsp. bolletii; $M$. chelonae and $M$. abscessus; $M$. intracellulare and M. chimaera; M. mucogenicum and M. phocaicum; subspecies of M. avium and M. abscessus; and species within the $M$. fortuitum group.

- Targeted gene sequencing may be required for the rare and novel species of NTM, particularly the slowly growing species as the database lacks the rare species.

\section{Author contributions}

JS Michael and V Balaji conceptualized the study. AS Sam, NKD Raghupathi and MM Ninan analyzed, interpreted data and wrote the manuscript. AS Sam and R Ranjani carried out bench work and generated data. JS Michael, MM Ninan and V Balaji critically revised and approved the manuscript. All authors read and approved the manuscript. 


\section{Acknowledgments}

M Gowri provided assistance with regards to the statistical tools to be used and M Dhivya provided technical assistance during the DNA extraction for sequencing. The approval for this study was provided by Institutional Review Board, Christian Medical College, Vellore.

Financial \& competing interests disclosure

This work was supported by the FLUID research grant of Christian Medical College Vellore (Institutional Review Board min no. 11224). The authors have no relevant affiliations or financial involvement with any organization or entity with a financial interest in or financial conflict with the subject matter or materials discussed in the manuscript. This includes employment, consultancies, honoraria, stock ownership or options, expert testimony, grants or patents received or pending, or royalties. The authors have no other relevant affiliations or financial involvement with any organization or entity with a financial interest in or financial conflict with the subject matter or materials discussed in the manuscript apart from those disclosed.

No writing assistance was utilized in the production of this manuscript.

\section{Ethical conduct of research}

The authors state that they have obtained appropriate institutional review board approval or have followed the principles outlined in the Declaration of Helsinki for all human or animal experimental investigations. In addition, for investigations involving human subjects, informed consent has been obtained from the participants involved.

\section{Informed consent disclosure}

The authors state that they have obtained verbal and written informed consent from the patient/patients for the inclusion of their medical and treatment history within this case report.

\section{Open access}

This work is licensed under the Creative Commons Attribution 4.0 License. To view a copy of this license, visit http://creativecommons.org/licenses/by/4.0/

\section{References}

Papers of special note have been highlighted as: $\bullet$ of interest

1. Taxonomy of mycobacteria. In: Mycobacteria. Goodfellow M, Magee JG (Eds). Springer Link, Berlin, Germany, 1-71 (1998).

2. LPSN. Mycobacterium (2020). www.bacterio.net/mycobacterium.html

3. Wolinsky E. Nontuberculous mycobacteria and associated diseases. Am. Rev. Respir. Dis. 119(1), 107-159 (1979).

4. Benson CA. Disease due to the Mycobacterium avium complex in patients with AIDS: epidemiology and clinical syndrome. Clin. Infect. Dis. 18(Suppl. 3), S218-S222 (1994).

5. Falkinham JO. Epidemiology of infection by nontuberculous mycobacteria. Clin. Microbiol. Rev. 9(2), 177-215 (1996).

6. Haworth CS, Banks J, Capstick T et al. British Thoracic Society guidelines for the management of Nontuberculous mycobacterial pulmonary disease (NTM-PD). Thorax 72(Suppl. 2), ii1-ii64 (2017).

7. bioMerieux. VITEK® MS Mass Spectrometry Microbial Identification System (2020). www.biomerieux-diagnostics.com/vitekr-ms-0

8. Luo L, Cao W, Chen W et al. Evaluation of the VITEK MS knowledge base version 3.0 for the identification of clinically relevant Mycobacterium species. Emerg. Microbes Infect. 7(1), 114 (2018).

9. Mediavilla-Gradolph MC, De Toro-Peinado I, Bermúdez-Ruiz MP et al. Use of MALDI-TOF MS for identification of nontuberculous mycobacterium species isolated from clinical specimens. BioMed Res. 2015, e854078 (2015).

10. Leyer C, Gregorowicz G, Mougari F, Raskine L, Cambau E, de Briel D. Comparison of Saramis 4.12 and IVD 3.0 Vitek MS matrix-assisted laser desorption ionization-time of flight mass spectrometry for identification of mycobacteria from solid and liquid culture media. J. Clin. Microbiol. 55(7), 2045-2054 (2017).

11. Bruker Daltonics. Overview Mycobacteria Identification (2020). www.bruker.com/products/mass-spectrometry-and-separations/ivd-ce -certified-maldi-biotyper/mycobacteria-identification/overview-mycobacteria-identification.html

12. Tortoli E. Impact of genotypic studies on mycobacterial taxonomy: the new mycobacteria of the 1990s. Clin. Microbiol. Rev. 16(2), 319-354 (2003).

13. Joao I, Cristovao P, Antunes L, Nunes B, Jordao L. Identification of nontuberculous mycobacteria by partial gene sequencing and public databases. Int. J. Mycobacteriol. 3(2), 144-151 (2014).

14. Slany M, Pavlik I. Molecular detection of nontuberculous mycobacteria: advantages and limits of a broad-range sequencing approach. J. Mol. Microbiol. Biotechnol. 22(4), 268-276 (2012). 
15. Roth A, Fischer M, Hamid ME, Michalke S, Ludwig W, Mauch H. Differentiation of phylogenetically related slowly growing mycobacteria based on 16S-23S rRNA gene internal transcribed spacer sequences. J. Clin. Microbiol. 36(1), 139-147 (1998).

16. Griffith DE, Aksamit T, Brown-Elliott BA et al. An official ATS/IDSA statement: diagnosis, treatment, and prevention of nontuberculous mycobacterial diseases. Am. J. Respir. Crit. Care Med. 175(4), 367-416 (2007).

17. Henkle E, Hedberg K, Schafer SD, Winthrop KL. Surveillance of extrapulmonary nontuberculous mycobacteria infections, Oregon, USA, 2007-2012. 23(10), 1627-1630 (2017).

18. Kham-Ngam I, Chetchotisakd P, Ananta P et al. Epidemiology of and risk factors for extrapulmonary nontuberculous mycobacterial infections in Northeast Thailand. PeerJ. 6, e5479 (2018).

19. U.S Food and Drug Administration. 510(k) substantial equivalence determination decision summary (2017). www.accessdata.fda.gov/cdrh_docs/reviews/K162950.pdf

20. Turner S, Pryer KM, Miao VP, Palmer JD. Investigating deep phylogenetic relationships among cyanobacteria and plastids by small subunit rRNA sequence analysis. J. Eukaryot. Microbiol. 46(4), 327-338 (1999).

21. Yu X, Lu L, Chen G et al. Identification and characterization of Nontuberculous mycobacteria isolated from tuberculosis suspects in Southern-central China. PloS ONE 9(12), e114353 (2014).

22. Jesudason MV, Gladstone P. Non tuberculous mycobacteria isolated from clinical specimens at a tertiary care hospital in South India. Indian J. Med. Microbiol. 23(3), 172-175 (2005).

23. Park SC, Kang MJ, Han CH et al. Prevalence, incidence, and mortality of nontuberculous mycobacterial infection in Korea: a nationwide population-based study. BMC Pulm. Med. 19(1), 140 (2019).

24. Narang R, Narang P, Mendiratta DK. Isolation and identification of nontuberculous mycobacteria from water and soil in central India. Indian J. Med. Microbiol. 27(3), 247 (2009).

25. Falkinham JO. Environmental sources of nontuberculous mycobacteria. Clin. Chest Med. 36(1), 35-41 (2015).

26. Joshi SC, Diwan V, Joshi R et al. "How can the patients remain safe, if we are not safe and protected from the infections"? a qualitative exploration among health-care workers about challenges of maintaining hospital cleanliness in a resource limited tertiary setting in rural India. Int. J. Environ. Res. Public. Health. 15(9), 1942 (2018).

27. Brode SK, Daley CL, Marras TK. The epidemiologic relationship between tuberculosis and Nontuberculous mycobacterial disease: a systematic review. Int. J. Tuberc. Lung Dis. Off. J. Int. Union Tuberc. Lung Dis. 18(11), 1370-1377 (2014).

28. Varley CD, Ku JH, Henkle E, Schafer SD, Winthrop KL. Disseminated nontuberculous mycobacteria in HIV-infected patients, Oregon, USA, 2007-2012. Emerg. Infect. Dis. 23(3), 533-535 (2017).

29. Dhar K, Shenoy VP, Vishwanath S, Prabhu M. Disseminated Mycobacterium avium intracellulare complex (MAC) disease in a retropositive patient caused by noncompliance of HAART. Ann. Trop. Med. Public Health. 9(3), 194 (2016).

30. Ding LW, Lai CC, Lee LN, Hsueh PR. Disease caused by nontuberculous mycobacteria in a university hospital in Taiwan, 1997-2003. Epidemiol. Infect. 134(5), 1060-1067 (2006).

31. Snydman DR, Redelman-Sidi G, Sepkowitz KA. Rapidly growing mycobacteria infection in patients with cancer. Clin. Infect. Dis. 51(4), 422-434 (2010).

32. Hoefsloot W, van Ingen J, Andrejak C et al. The geographic diversity of nontuberculous mycobacteria isolated from pulmonary samples: an NTM-NET collaborative study. Eur. Respir. J. 42(6), 1604-1613 (2013).

33. Umrao J, Singh D, Zia A et al. Prevalence and species spectrum of both pulmonary and extrapulmonary nontuberculous mycobacteria isolates at a tertiary care center. Int. J. Mycobacteriol. 5(3), 288-293 (2016).

34. MM18: DNA Target Sequencing \& Bacteria \& Fungi ID. In: Interpretive Criteria for Identification of Bacteria and Fungi by Targeted DNA Sequencing 2nd Edition Clinical Laboratory Standards Institute, PA, USA (2018).

35. Thegerström J, Jönsson B, Brudin L et al. Mycobacterium avium Subsp. avium and Subsp. hominissuis give different cytokine responses after in vitro stimulation of human blood mononuclear cells. PLoS One 7(4), e34391 (2012).

36. Brown-Elliott BA, Fritsche TR, Olson BJ et al. Comparison of two commercial matrix-assisted laser desorption/ionization-time of flight mass spectrometry (MALDI-TOF MS) systems for identification of nontuberculous mycobacteria. Am. J. Clin. Pathol. 152(4), 527-536 (2019).

- Article compares the two matrix-assisted laser desorption ionization-time of flight MS platforms for nontuberculous mycobacteria identification.

37. Buckwalter SP, Olson SL, Connelly BJ et al. Evaluation of matrix-assisted laser desorption ionization-time of flight mass spectrometry for identification of mycobacterium species, nocardia species, and other aerobic actinomycetes. J. Clin. Microbiol. 54(2), 376-384 (2016).

38. US FDA. Communication with bioMerieux, Inc. (2018). www.accessdata.fda.gov/cdrh_docs/pdf18/K181412.pdf

39. Jorgensen JH, Pfaller MA, Carroll KC. Manual of Clinical Microbiology. ASM Press, Washington DC, USA (2015). 